\title{
Diabetes insípida de origen central secundario a hipofisitis. Reporte de 2 casos
}

\section{Central diabetes insipidus secondary to hypophysitis. Report of two cases}

\author{
Tovar $H,{ }^{1}$ Flórez A, ${ }^{1}$ Quintero $G,{ }^{2}$ Concha $D .{ }^{3}$
}

\author{
${ }^{1}$ Médico internista, endocrinólogo, Hospital San José. Bogotá, \\ Colombia. \\ ${ }^{2}$ Residente de medicina interna, Fundación Universitaria de \\ Ciencias de la Salud. Bogotá, Colombia. \\ ${ }^{3}$ Médico internista, fellow de endocrinología, Fundación \\ Universitaria de Ciencias de la Salud. Bogotá, Colombia. \\ Autor de correspondencia: Andrés Flórez Romero \\ Correo electrónico: andresflorez25@hotmail.com \\ Fecha de recepción: 9/02/2020 \\ Fecha de aceptación: 18/09/2020
}

\section{Resumen}

La diabetes insípida es una entidad poco frecuente caracterizada por una deficiencia en la secreción de la hormona antidiurética (ADH) o resistencia a su acción, lo que genera una alteración para concentrar la diuresis y, en consecuencia, ocasiona altos volúmenes de orina diluida. La diabetes insípida puede ser de origen renal o central, y esta última es la más frecuente. La poliuria y polidipsia son las expresiones clínicas predominantes en respuesta a la incapacidad del paciente de mantener un equilibrio hídrico. Las causas de la diabetes insípida central son diversas, tales como después de la cirugía pituitaria, adenoma hipofisario, encefalopatía isquémica, trauma, hipofisitis, origen genético o idiopática. La hipofisitis primaria es una entidad rara, caracterizada por un proceso inflamatorio de la glándula hipofisaria, que puede comprometer la función de la hipófisis anterior o posterior. A continuación, se presentan 2 casos de pacientes con diabetes insípida secundaria a hipofisitis estudiadas ambulatoriamente y de manera intrahospitalaria.

Palabras clave: diabetes insípida, hipofisitis, arginina vasopresina.

\begin{abstract}
Diabetes insipidus is a rare entity characterized by a deficiency in the secretion of the antidiuretic hormone (ADH) or resistance to its action, which causes an alteration in concentrating diuresis, consequently, high volumes of diluted urine. Diabetes insipidus can be of renal or central origin and the latter is the most frequent. Polyuria and polydipsia are the predominant clinical expressions in response to the patient's inability to maintain a water balance. The causes of central diabetes insipidus are diverse, such as after pituitary surgery, pituitary adenoma, ischemic encephalopathy, trauma, hypophysitis, genetic or idiopathic origin. Primary hypophysitis is a rare entity, characterized by an inflammatory process of the pituitary gland, that affects the anterior and/or posterior pituitary function. We presented two cases of patients with diabetes insipidus secondary to hypophysitis studied on outpatient and inpatient.
\end{abstract}

Keywords: Diabetes Insipidus; Hypophysitis; Arginine Vasopressin.

\section{Introducción}

La diabetes insípida es una enfermedad rara con una prevalencia reportada de 1:25 000 personas (1). Es el resultado final de múltiples condiciones que afectan el sistema hipotálamo-neurohipofisario (2). Dentro de las causas poco frecuentes se encuentra la hipofisitis, proceso inflamatorio de la hipófisis que puede ser de origen primario o secundaria a patologías autoinmunes o infiltrativas con compromiso multisistémico (3). La incidencia de la hipofisitis no es clara, pero se estima que es de 1 caso por cada 9 millones de personas (4). A continuación, se presentan 2 casos de pacientes con diagnóstico de diabetes insípida secundaria a hipofisitis. 


\section{Presentación de los casos}

\section{Paciente 1}

Paciente femenina de 31 años, sin antecedentes patológicos, quien consultó al servicio de endocrinología del Hospital de San José (Bogotá, Colombia) por un cuadro clínico de 5 meses de evolución caracterizado por polidipsia (ingesta de 15 litros de agua al día), asociado con poliuria (gasto urinario de 4,8 mL/ $\mathrm{kg} / \mathrm{h}$ ). La paciente refería adicionalmente cefalea, galactorrea bilateral, acné y amenorrea de un año de evolución. Se descartaron causas secundarias de poliuria como síndrome de Cushing, medicamentos o alteraciones del potasio o del calcio.

Dada la persistencia de la sintomatología, se realizó una prueba de restricción hídrica (Tabla 1) compatible con diabetes insípida de origen central. Se solicitó una imagen de resonancia magnética (IRM) con gadolinio sugestiva de hipofisitis (Figura 1 A y B). Se inició el manejo con desmopresina $60 \mu$ g sublingual cada 12 horas y presentó mejoría de los síntomas. Se descartaron causas infecciosas (serología VDRL no reactiva, virus de la inmunodeficiencia humana [VIH] negativo, sin clínica de tuberculosis o bacteriemia) y autoinmunes (anticuerpos antinucleares [ANA] por inmunofluorescencia indirecta $[\mathrm{IFI}]<1 / 40$ diluciones, sin clínica de enfermedad autoinmune asociada), o hipofisitis asociada a inmunoglobulina G 4 (IgG4) (inmunoglobulina G [IgG]: $820 \mathrm{mg} / \mathrm{dL}$, valor de referencia [VR]: 650-1600 mg/dL; IgG4: $12 \mathrm{mg} / \mathrm{dL}$, VR: 4-86 mg/dL; inmunoglobulina M [IgM]: $110 \mathrm{mg} / \mathrm{dL}$, VR: 54$300 \mathrm{mg} / \mathrm{dL}$; inmunoglobulina A [IgA]: $120 \mathrm{mg} / \mathrm{dL}$, VR: 40-350 $\mathrm{mg} / \mathrm{dL}$ ). Los estudios complementarios de hipófisis anterior, a excepción de la prolactina, estuvieron dentro de los límites normales (factor de crecimiento insulínico tipo 1 [IGF-1] 112 ng/mL, VR: 88 -537 ng/mL; prolactina 32,11 ng/mL, VR: 5,1826,53 ng/mL; cortisol sérico: 18,50 $\mu \mathrm{g} / \mathrm{dL}$, VR: 3,7-19,4 $\mu \mathrm{g} /$ $\mathrm{dL}$; y tirotropina [TSH]: 0,88 $\mu \mathrm{UI} / \mathrm{mL}, \mathrm{VR}: 0,46-4,68 \mu \mathrm{UI} / \mathrm{mL}$; tiroxina $\left[\mathrm{T}_{4}\right]$ libre normal). Debido a la resolución espontánea de la galactorrea con la reaparición de ciclos menstruales y el seguimiento de la prolactina con normalización en los niveles, no requirió manejo con agonistas de dopamina.

Se realizó una prueba de restricción hídrica (Tabla 1), cuando presentó hipernatremia (148 mmol/L), se suspendió la prueba y se administró desmopresina $1 \mu \mathrm{g}$ subcutánea (SC) con aumento de osmolalidad urinaria hasta 220 m0sm/kg (150 \% por encima del valor basal). De acuerdo con estos hallazgos, se consideró una diabetes insípida de origen central completa.

\section{Paciente 2}

Paciente masculino de 31 años que consultó al servicio de endocrinología por un cuadro clínico de 3 meses de evolución consistente en polidipsia (ingesta de 10-12 litros de agua al día), poliuria (gasto urinario de $4 \mathrm{~mL} / \mathrm{kg} /$ hora) y nicturia. Asociado con el cuadro, refirió cefalea sin signos de alarma de 12 meses de evolución. Le habían indicado un manejo empírico con desmopresina inhalada un puff cada 12 horas, con mejoría del gasto urinario. Acudió con paraclínicos que mostraron una densidad urinaria en 1,005 y sodio sérico normal (143 mEq/L), realizados con suspensión de desmopresina por 18 horas, sin restricción hídrica. Fue valorado previamente por psiquiatría sin patología asociada. Se indicó la toma de osmolalidad urinaria, vasopresina e IRM de silla turca de forma ambulatoria, con ayuno previo de 6 horas y suspensión de la desmopresina por 24 horas.

Los resultados evidenciaron una osmolalidad urinaria menor de $300 \mathrm{mOsm} / \mathrm{kg}$ (resultado en $222 \mathrm{mOsm} / \mathrm{kg}$ ) y hormona antidiurética $<2 \mathrm{pg} / \mathrm{mL}$ (resultado en $1 \mathrm{pg} / \mathrm{mL}$ ), con gasto urinario de 4,3 mL/kg/h (volumen urinario: $8300 \mathrm{~mL} / 24$ horas), por lo cual se confirmó la diabetes insípida. Se descartaron causas secundarias de poliuria. En la IRM de silla turca con gadolinio (Figura 1) se observó una imagen compatible con microadenoma (Figura $\mathbf{1}$ C), con aumento del tamaño

Tabla 1. Prueba de restricción hídrica, caso 1

\begin{tabular}{l|c|c|c|c|c}
\hline \multirow{2}{*}{ Hora } & Sodio (mmol/L) & OsmP (mOsm/kg) & OsmU (mOsm/kg) & Peso (kg) & PA (mm Hg) \\
\hline 0 & 140 & 268 & 58,1 & 70,3 & $123 / 71$ \\
\hline 1 & 145 & 278,5 & 77,1 & 68,6 & $120 / 70$ \\
\hline 2 & 146 & 280,3 & 82,7 & 68,3 & $126 / 70$ \\
\hline 3 & 148 & 283 & 77,3 & 68 & $120 / 80$ \\
\hline 2 h pos-DMP & & & 220 & & \\
\hline
\end{tabular}

DMP: desmopresina, OsmP: osmolalidad plasmática, OsmU: osmolalidad urinaria, PA: presión arterial. 
en hemihipófisis izquierda y desviación del tallo hipofisario a la derecha, con una imagen pseudotriangular del infundíbulo (Figura 1 D) y ausencia de hiperintensidad en T1 de la hipófisis posterior. Se consideró un cuadro de hipofisitis con diabetes insípida central. Teniendo en cuenta el antecedente de patología rinosinusal, se inició la terapia con desmopresina orodispersable, con titulación de dosis hasta $120 \mu \mathrm{g}$ cada 12 horas, y se presentó mejoría del gasto urinario y la polidipsia. Los estudios complementarios hipofisarios descartaron funcionalidad del microadenoma (IGF-1: $160 \mathrm{ng} / \mathrm{mL}$, VR:

Figura 1. IRM con gadolinio proyección T1. A. Paciente 1: imagen coronal, hiperintensidad homogénea de la hipófisis, con aumento del volumen y extensión supraselar (flecha blanca). B. Paciente 1: imagen sagital, ausencia de hiperintensidad en la hipófisis posterior (flecha blanca). C. Paciente 2: imagen coronal, aumento del volumen hipofisario izquierdo con adenoma de 9 × $6 \mathrm{~mm}$ (flecha blanca) y desviación del tallo hipofisario a la derecha. D. Paciente 2: imagen sagital, hiperintensidad del tallo hipofisario (flecha blanca) con apariencia pseudotriangular (infundibulitis) y ausencia de hiperintensidad de la hipófisis posterior.

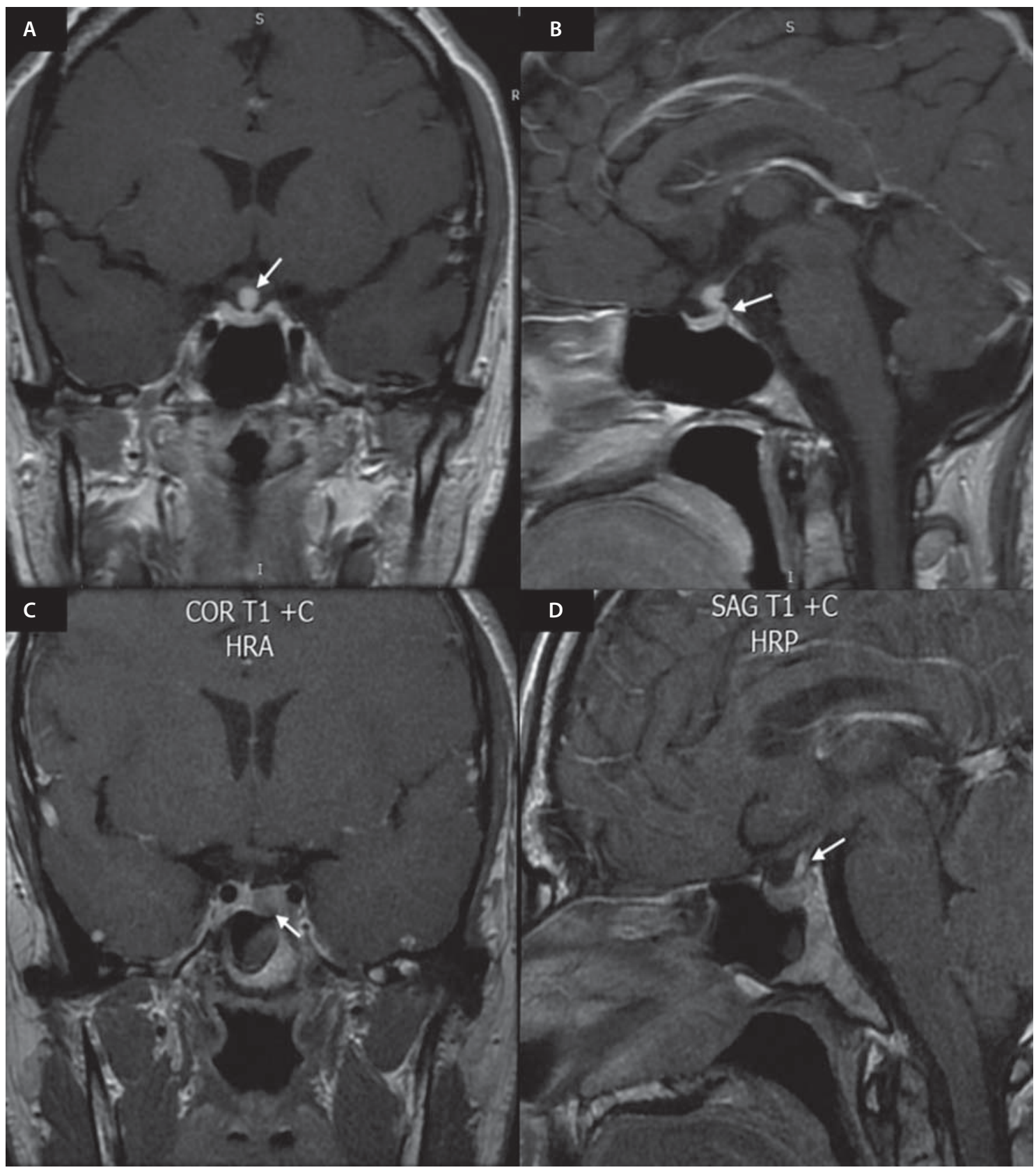


71,2-234; prolactina: 11,4 ng/mL, VR: 4,04-15,2; cortisol a. m.: 10,2 $\mu \mathrm{g} / \mathrm{dL}$, VR: 4,82-19,5; TSH: 1,77 $\mu \mathrm{UI} / \mathrm{mL}$, VR: 0,27-4,2; $\mathrm{T}_{4}$ libre: 1,39 ng/dL, VR: 0,93-1,7). El paciente no tiene síntomas o alteraciones en los paraclínicos sugestivos de enfermedad autoinmune, granulomatosa o infecciosa que sugieran causas secundarias de hipofisitis.

\section{Discusión}

La diabetes insípida pertenece a un grupo de enfermedades caracterizadas por poliuria y polidipsia, cuyo origen puede ser hereditario o adquirido (5). La poliuria es uno de los síntomas cardinales de este síndrome; en adultos se define como un volumen de orina $>3$ litros en 24 horas o $>40 \mathrm{~mL} / \mathrm{kg} /$ día, o un gasto urinario $>2 \mathrm{~mL} / \mathrm{kg} / \mathrm{h}$. La polidipsia es consecuencia del aumento inicial del sodio sérico y la osmolaridad (6). La nicturia e incluso la enuresis también se pueden presentar en estos pacientes. Estos síntomas fueron referidos en los 2 casos referenciados. Una vez confirmada la poliuria, la evidencia de osmolalidad urinaria disminuida (<300 m0sm $/ \mathrm{kg}$ ) aumenta la sospecha diagnóstica. Existen otras entidades que presentan poliuria dentro de las cuales está la diabetes mellitus tipo 2 mal controlada (asociada con glucosuria), polidipsia primaria, síndrome de Cushing, medicamentos (p. ej. diuréticos), hipercalcemia e hipopotasemia. La polidipsia primaria es uno de los diagnósticos diferenciales más importantes; en este caso, la poliuria es una respuesta apropiada en relación con una mayor ingesta de agua (7).

La diabetes insípida es considerada una enfermedad poco frecuente con una prevalencia de 1:25000 personas (1). Existen múltiples condiciones que afectan la neurohipófisis o el hipotálamo y generan una síntesis deficiente de hormona antidiurética (ADH) (5). El diagnóstico se puede realizar de acuerdo con los siguientes contextos clínicos: si hay evidencia de hipernatremia, es suficiente con observar un incremento del $50 \%$ de la osmolalidad urinaria con una prueba de desmopresina subcutánea $(1 \mathrm{mg})$. En caso de normonatremia, la prueba de restricción hídrica realizada de forma intrahospitalaria es la más utilizada y tiene en cuenta la capacidad fisiológica de concentrar la orina y la respuesta con el uso de desmopresina. Esta prueba fue la que se le realizó a la paciente 1. En la Figura 2 se incluye el algoritmo de interpretación utilizado por nuestro servicio para la realización de la prueba de restricción hídrica.

También se puede realizar la prueba de forma ambulatoria con medición de la $\mathrm{ADH}$ en el contexto de una osmolalidad urinaria disminuida (<300 m0sm/kg) e ingesta hídrica a voluntad. Los niveles de $\mathrm{ADH}>2 \mathrm{pg} / \mathrm{mL}$ sugieren diabetes insípida nefrogénica, mientras que los niveles de ADH $<1 \mathrm{pg} /$ $\mathrm{mL}$ y la pérdida de la hiperintensidad de la hipófisis posterior en T1 sugieren diabetes insípida central (1). Por una parte, se debe tener en cuenta que la hiperintensidad en T1 de la hipófisis posterior puede estar ausente en el $20 \%$ de las personas sanas (8) y en el $39 \%$ de los pacientes con polidipsia primaria (9). Por otra parte, la medición de la ADH presenta dificultades técnicas en la toma y una alta variabilidad interanalítica. Este método diagnóstico fue escogido en el paciente 2 , teniendo en cuenta la intensidad de los síntomas, la respuesta previa en el control de la polidipsia y poliuria con uso de desmopresina, y la ausencia de síntomas psiquiátricos que sugirieran polidipsia primaria; y se consideró el diagnóstico con base en la osmolalidad urinaria disminuida con un nivel límite de la ADH (1 pg/mL) y los hallazgos en la IRM.

Posiblemente, la copeptina (proteína derivada de la preprovasopresina), aún no disponible en Colombia, sea más útil para el diagnóstico de la diabetes insípida (10), teniendo en cuenta su excelente correlación con los niveles de la ADH y la osmolaridad plasmática, y una mayor vida media que la $\mathrm{ADH}$. Recientemente, Fenske y colaboradores (9) compararon en 141 pacientes la prueba de restricción hídrica con un estudio que consistía en la infusión de solución hipertónica hasta obtener una concentración de sodio sérico de $150 \mathrm{mmol} / \mathrm{L}$ y la medición de copeptina después de conseguir este objetivo. Se consideró la diabetes insípida en caso de niveles de copeptina $<4,9 \mathrm{pmol} / \mathrm{L}$ y polidipsia primaria con niveles mayores a este punto de corte. La prueba de solución hipertónica con medición de copeptina demostró ser más precisa que la de restricción hídrica para diferenciar la polidipsia primaria de la diabetes insípida; la sensibilidad y especificidad para el punto de corte de 4,9 pmol/L fue del 93,2 \% y $100 \%$, respectivamente.

El objetivo del tratamiento de la diabetes insípida es controlar la poliuria. Los análogos de la vasopresina (1-deamino8-D-arginina vasopresina [DDAVP]) son el tratamiento de elección (11). Se administran por vía intranasal, oral o parenteral. La vida media, absorción e inicio de acción varían en cada una (12). La elección de la vía de administración depende de las condiciones del paciente: la patología rinosinusal limita la administración intranasal, por lo que en estos casos se prefiere la vía oral, como en el paciente 2 .

Aunque la etiología de la diabetes insípida en muchos casos es desconocida, este síndrome puede ser secundario a trauma craneoencefálico (TCE), cirugía hipotálamo-hipofisaria, enfermedades vasculares, enfermedades infiltrativas como la histiocitosis de Langerhans o la sarcoidosis, tumores como el germinoma o craneofaringioma, metástasis o hipofisitis (13). En ambos pacientes se consideró la asociación de diabetes insípida con hipofisitis dados los hallazgos imagenológicos y teniendo en cuenta que se descartaron las otras causas referidas.

La hipofisitis se caracteriza por la inflamación de la glándula pituitaria, que puede presentarse tanto en la hipófisis anterior como en la posterior. El compromiso en la función hipofisaria depende del segmento de la hipófisis afectada. Cuando se asocia con diabetes insípida se observa una alteración especialmente del infundíbulo y la neurohipófisis (14), término 
Figura 2. Algoritmo de la prueba de restricción hídrica.

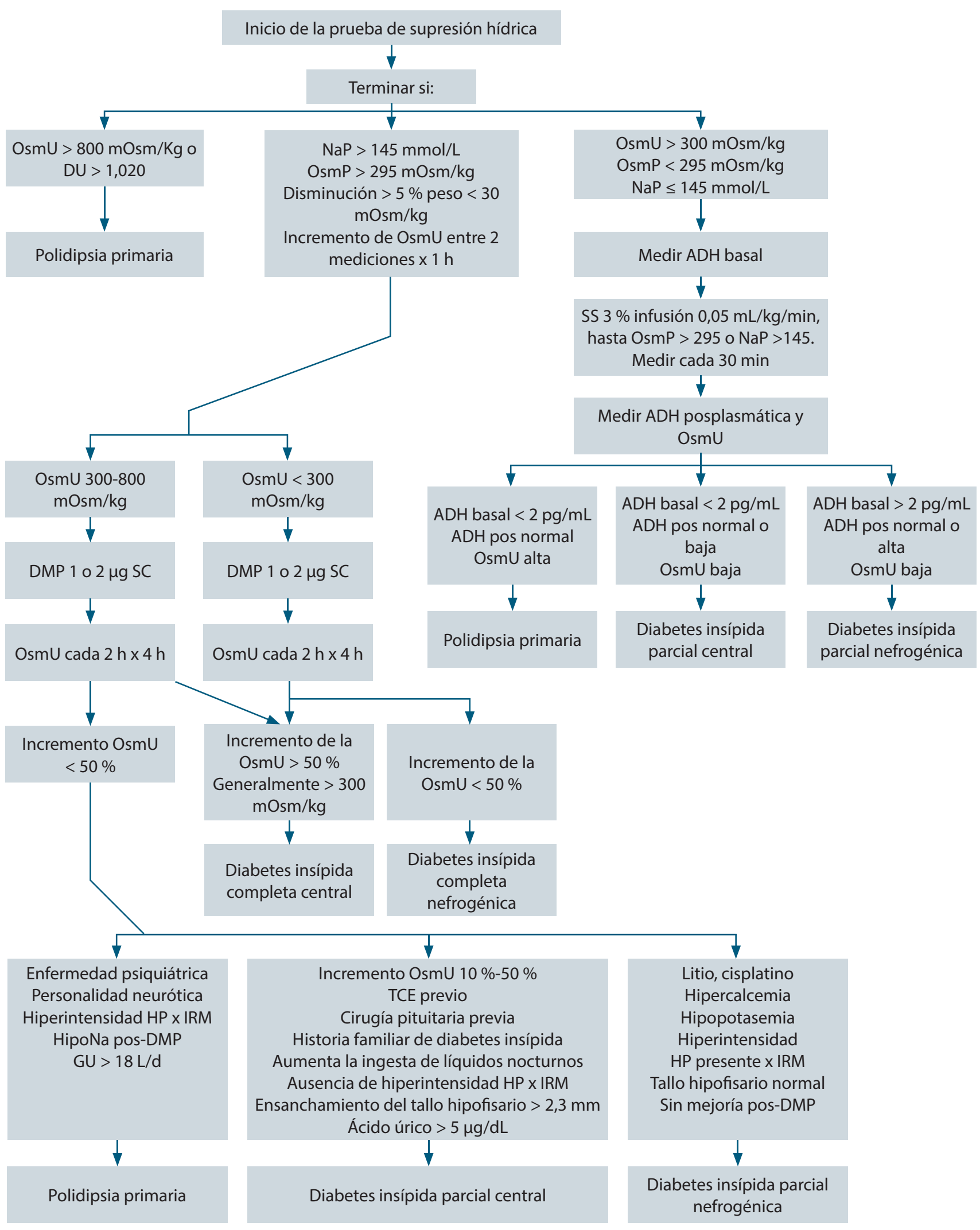

DU: densidad urinaria; GU: gasto urinario; HP: hipófisis posterior; NaP: sodio plasmático; IRM: imagen de resonancia magnética de silla turca, SS: solución salina; TCE: trauma craneoencefálico. 
conocido como infundíbulo-neurohipofisitis. La hipofisitis se clasifica como primaria o secundaria a condiciones inflamatorias multisistémicas (p. ej. lupus eritematoso sistémico [LES] y síndrome poliglandular).

Tiene una incidencia anual estimada de 1 caso por cada 9 millones de personas y una prevalencia de 0,22 \%-0,88 \%, que afecta a hombres y mujeres en igual proporción $(3,4)$. Los síntomas más frecuentes de la hipofisitis en la fase crónica son la cefalea y el aumento de peso, que presentan una asociación con la diabetes insípida aproximadamente en un $54 \%$ de los casos (15). La hipofisitis se puede clasificar según la localización del compromiso hipofisario en adenohipofisitis (hiperplasia de la hipófisis anterior sin lesión focal, sin compromiso de la neurohipófisis), infundíbulo-neurohipofisitis (alteración en el tallo hipofisario, el infundíbulo y la neurohipófisis) y panhipofisitis (compromiso adenopituitario, neurohipófisis, del tallo pituitario y del infundíbulo) (16).

Los hallazgos característicos en la IRM cerebral son la hiperintensidad homogénea de la hipófisis anterior, con aumento del volumen hipofisario de predominio supraselar e hiperintensidad de la duramadre adyacente (signo del tallo dural). En caso de infundibulitis, se puede observar hiperintensidad con apariencia pseudotriangular del infundíbulo y pérdida de la hiperintensidad de la hipófisis posterior en T1 cuando se asocia con diabetes insípida $(3,16,17)$, tal como se describe en los dos presentes casos. Estos signos son más frecuentes en la hipofisitis linfocítica clásica. El compromiso aislado del tallo hipofisario es más común en la hipofisitis linfocítica, histiocitosis de Langerhans, tumores de células germinales o metástasis (18). Los hallazgos de silla turca vacía pueden encontrarse como resultado del proceso inflamatorio crónico.

La variante más frecuente es la hipofisitis linfocítica (76 \%$86 \%$ de los casos) (19), cuyo diagnóstico solo puede ser confirmado de forma histológica, y se puede presentar en el tercer trimestre del embarazo o en el posparto. La hiperprolactinemia es característica en la fase aguda (como ocurrió en la paciente 1), pero el compromiso de la hipófisis anterior se presenta en el siguiente orden de frecuencia: corticotropina (ACTH), gonadotropinas, TSH y hormona del crecimiento (GH) (18). Otros tipos de hipofisitis descritos son la hipofisitis xantomatosa (alrededor de 27 casos reportados) y necrotizante $(0,6 \%$ de las causas de hipofisitis). La hipofisitis xantomatosa se ha considerado una condición inflamatoria probablemente desencadenada por la ruptura de un quiste de Rathke. La hipofisitis granulomatosa puede considerarse primaria o secundaria a otras patologías. También se han descrito formas mixtas de tipo linfogranulomatosa y xantogranulomatosa. Todas estas formas de hipofisitis requieren un estudio histológico para su confirmación (19).

Las enfermedades granulomatosas constituyen una causa importante de hipofisitis a tener en cuenta. Los granulomas a nivel hipofisario pueden estar asociados con histiocitosis de Langerhans, sarcoidosis, sífilis, tuberculosis hemocromatosis o poliangitis con granulomatosis. Incluso, se ha descrito hipofisitis asociada con VIH o secundaria a infecciones en la hipófisis originadas por diseminación hematológica $(18,19)$. También se han descrito casos de hipofisitis asociada con enfermedades autoinmunes como LES, síndrome poliglandular, tiroiditis de Hashimoto y la anemia perniciosa $(20,21)$. Dada la asociación con condiciones sistémicas o infiltrativas, es importante considerar estas enfermedades de acuerdo con las manifestaciones clínicas y realizar los estudios pulmonares, óseos, infecciosos o autoinmunes pertinentes (18).

Se ha descrito la hipofisitis relacionada con IgG4 (que corresponde a $<5 \%$ de los casos de hipofisitis), la cual se presenta de forma aislada o, en el 60\%-90 \% de los casos, asociada con el compromiso multisistémico propio de esta enfermedad (páncreas, retroperitoneo, pulmones y riñones). Es más prevalente en Asia, con una edad de presentación cercana a los 55 años; se asocia generalmente con el compromiso de hipófisis anterior y la diabetes insípida. Para el diagnóstico se tienen en cuenta los criterios de Leporati, que parten de la identificación de niveles elevados de IgG4 en el tejido hipofisario, la sangre u otro órgano; y de la respuesta a los corticoides (22).

Recientemente, se han descrito casos de hipofisitis asociada con los medicamentos utilizados para inmunoterapia en cáncer como ipilimumab (anticuerpo monoclonal de tipo inmunoglobulina G1-kappa [IgG1к] frente al antígeno 4 del linfocito T citotóxico [CTLA-4]) o nivolumab (anticuerpo monoclonal contra el ligando 1 de muerte programada [PD-1]) (23).

De acuerdo con lo anterior, teniendo en cuenta la ausencia de manifestaciones de enfermedad sistémicas de tipo granulomatoso y debido a la evidencia del perfil autoinmune normal sin síntomas de enfermedades autoinmunes y los niveles de IgG4 normales, se consideró que nuestros pacientes posiblemente cursaron con hipofisitis linfocítica. El manejo depende de la deficiencia hormonal, realizando una evaluación amplia de todas las hormonas hipofisarias y un reemplazo acorde con los hallazgos. La cirugía solo está indicada en caso de compromiso de la vía visual o en déficit neurológico secundario a efecto compresivo. El uso de corticoides muestra una respuesta inicial, pero con una frecuencia de recaída de hasta el 38 \% (15) y los conocidos efectos secundarios asociados con su uso crónico; por estos motivos no se consideraron en nuestros pacientes.

\section{Conclusión}

La diabetes insípida siempre debe considerarse dentro del diagnóstico diferencial de poliuria. En este artículo se presentan 2 pacientes con esta patología secundaria a hipofisitis, causa poco frecuente con buena respuesta al manejo farmacológico. Son necesarios estudios adicionales para evaluar el impacto de los corticoides a largo plazo en casos de hipofisitis linfocítica. 


\section{Agradecimientos}

Agradecemos a los pacientes que permitieron acceder a la información personal, la historia clínica e imágenes diagnósticas.

\section{Consideraciones éticas}

De acuerdo con la resolución n. ${ }^{\circ} 008430$ de 1993, este es un estudio sin riesgo. Se realizó el consentimiento informado para la publicación de las imágenes y laboratorios incluidos en este reporte de caso.

\section{Conflictos de interés}

Los autores declaran no tener ningún conflicto de intereses.

\section{Referencias}

1. Robertson GL. Diabetes insipidus: Differential diagnosis and management. Best Pract Res Clin Endocrinol Metab. 2016;30(2):205-18.

2. Di Iorgi N, Napoli F, Allegri AE, Olivieri I, Bertelli E, Gallizia A, et al. Diabetes insipidus--diagnosis and management. Horm Res Paediatr. 2012;77(2):69-84.

3. Bellastella G, Maiorino MI, Bizzarro A, Giugliano D, Esposito K, Bellastella A, et al. Revisitation of autoimmune hypophysitis: knowledge and uncertainties on pathophysiological and clinical aspects. Pituitary. 2016;19(6):625-642.

4. Caturegli P, Newschaffer C, Olivi A, Pomper MG, Burger PC, Rose NR. Autoimmune hypophysitis. Endocr Rev. 2005;26(5):599-614.

5. Fenske W, Allolio B. Clinical review: Current state and future perspectives in the diagnosis of diabetes insipidus: a clinical review. J Clin Endocrino Metab. 2012;97(10):3426-37.

6. Knepper MA. Molecular physiology of urinary concentrating mechanism regulation of aquaporin water channels by vasopressin. Am J Physiol. 1997;272(1 Pt 2):F3-12.

7. Sailer C, Winzeler B, Christ-Crain M. Primary polydipsia in the medical and psychiatric patient: characteristics, complications and therapy. Swiss Med Wkly. 2017;147:w14514

8. Fujisawa I. Magnetic resonance imaging of the hypothalamic-neurohypophyseal system. J Neuroendocrinol. 2004;16(4):297-302.

9. Fenske W, Refardt J, Chifu I, Schnyder I, Winzeler B, Drummond J, et al. A Copeptin-Based Approach in the Diagnosis of Diabetes Insipidus. N Engl J Med. 2018;379(5):428-439.

10. Christ-Crain M. EJE AWARD 2019: New diagnostic approaches for patients with polyuria polydipsia syndrome. Eur J Endocrinol. 2019;181(1):R11 R21.

11. Qureshi S, Galiveeti S, Bichet DG, Roth J. Diabetes insipidus: celebrating a century of vasopressin therapy. Endocrinology. 2014;155(12):4605-21.

12. Oiso Y, Robertson GL, Nørgaard JP, Juul KV. Clinical review: Treatment of neurohypophyseal diabetes insipidus. J Clin Endocrinol Metab. 2013;98(10):3958-67.
13. Di Iorgi N, Allegri AE, Napoli F, Calcagno A, Calandra E, Fratangeli N, et al Central diabetes insipidus in children and young adults: etiological diagnosis and long-term outcome of idiopathic cases. J Clin Endocrinol Metab. 2014;99(4):1264-72.

14. Rivera JA. Lymphocytic hypophysitis: disease spectrum and approach to diagnosis and therapy. Pituitary. 2006;9(1):35-45.

15. Honegger J, Schlaffer S, Menzel C, Droste M, Werner S, Elbelt U, et al. Diagnosis of Primary Hypophysitis in Germany. J Clin Endocrinol Metab. 2015;100(10):3841-9.

16. Tartaglione T, Chiloiro S, Laino ME, Giampietro A, Gaudino S, Zoli A, et al Neuro-radiological features can predict hypopituitarism in primary autoimmune hypophysitis. Pituitary. 2018;21(4):414-424.

17. Koshiyama H, Sato H, Yorita S, Koh T, Kanatsuna T, Nishimura K, et al. Lymphocytic hypophysitis presenting with diabetes insipidus: case report and literature review. Endocr J. 1994;41(1):93-7.

18. Joshi MN, Whitelaw BC, Carroll PV. Mechanisms in endocrinology: Hypophysitis: diagnosis and treatment. Eur J Endocrinol. 2018;179(3):R151R163.

19. Angelousi A, Alexandraki K, Tsoli M, Kaltsas G, Kassi E. Hypophysitis (Including IgG4 and Immunotherapy). Neuroendocrinology. 2020;110(910):822-835

20. Thodou E, Asa SL, Kontogeorgos G, Kovacs K, Horvath E, Ezzat S. Clinica case seminar: lymphocytic hypophysitis: clinicopathological findings. J Clin Endocrinol Metab. 1995;80(8):2302-11.

21. Hashimoto K, Asaba K, Tamura K, Takao T, Nakamura T. A case of lymphocytic infundibuloneurohypophysitis associated with systemic lupus erythematosus. Endocr J. 2002;49(6):605-10.

22. Lojou M, Bonneville JF, Ebbo M, Schleinitz N, Castinetti F. IgG4 hypophysitis: Diagnosis and management. Presse Med. 2020;49(1):104016.

23. Faje A. Immunotherapy and hypophysitis: clinical presentation, treatment and biologic insights. Pituitary. 2016;19(1):82-92. 